\title{
Kernos
}

Revue internationale et pluridisciplinaire de religion grecque antique

1 | 1988

Varia

\section{Images et piété en Grèce classique : la contribution de l'iconographie céramique à l'étude de la religion grecque}

\section{Annie Verbanck-Piérard}

\section{(2) OpenEdition}

Journals

Édition électronique

URL : http://journals.openedition.org/kernos/118

DOI : $10.4000 /$ kernos. 118

ISSN : 2034-7871

Éditeur

Centre international d'étude de la religion grecque antique

Édition imprimée

Date de publication : 1 janvier 1988

ISSN : 0776-3824

Référence électronique

Annie Verbanck-Piérard, « Images et piété en Grèce classique : la contribution de l'iconographie céramique à l'étude de la religion grecque », Kernos [En ligne], 1 | 1988, mis en ligne le 31 janvier 2011, consulté le 30 avril 2019. URL : http://journals.openedition.org/kernos/118; DOI : 10.4000/kernos.118 
Kernos, 1(1988), p. 223-234.

\section{IMAGES ET PIÉTÉ EN GRÈCE CLASSIQUE : LA CONTRIBUTION DE L'ICONOGRAPHIE CÉRAMIQUE A L'ÉTUDE DE LA RELIGION GRECQUE}

Parmi les multiples disciplines qui, chacune, projettent un éclairage particulier sur la religion grecque antique et dont la rencontre permet d'entrevoir un système polythéiste d'une remarquable complexité, l'iconographie constitue indéniablement un champ d'investigation du plus haut intérêt et suscite des études diversifiées, de plus en plus nombreuses, où s'élabore progressivement une méthodologie autonome. Il serait donc utile, pour répondre au thème général du colloque, de résumer, à l'aide d'un bref panorama de la bibliographie récente, quels peuvent être les principaux apports des sources iconographiques à l'étude de la religion grecque. La plupart des données reprises ici sont déjà bien connues et largement débattues, comme le prouvent plusieurs colloques récents ${ }^{1}$. Aussi la présente synthèse n'a-t-elle pour autre but que de fournir une introduction sommaire et provisoire aux réunions ultérieures que le C.E.R.G.A. consacrerait à ce thème.

Dans les limites de cet exposé, il est impossible de s'attacher au statut général de l'image dans la religion grecque : c'est là un sujet immense, qui englobe toutes les formes d'art et d'artisanat : la

ABREVIATIONS

ABV : J.D. BEAZLEY, Attic Black-Figure Vase-Painters, Oxford, 1956.

ARV 2 : J.D. BEAZLEY, Attic Red-Figure Vase-Painters, 2e éd., Oxford, 1963. Cité des images: C. BERARD et al., La cité des images. Religion et société en Grèce antique, Lausanne-Paris, 1984. LIMC : Lexicon Iconographicum Mythologiae Classicae, Zurich, depuis 1981.

1 Par exemple : H. METZGER, 'EILSAOHOIIA. Actes du Colloque sur les problèmes de l'image dans le monde méditerranéen classique, Lourmarin, 2-3 septembre 1982, Rome, 1985; F. LISS ARRAGUE et F. THELAMON, Image et céramique grecque. Actes du Colloque de Rouen, 25-26 novembre 1982, Rouen, 1983; C. BÉRARD, Image et société en Grèce ancienne. L'iconographie comme méthode d'analyse. Actes du Colloque de Lausanne, 8-11 février 1984, Lausanne, 1987 (Cahiers d'Archéologie romande, 36). 
grande sculpture, les reliefs en pierre, terre cuite ou métal, la coroplathie, la toreutique, la céramique..., ainsi que les témoignages d'auteurs anciens évoquant, directement ou non, le rôle des images dans leur société 2 . Les quelques observations proposées ici se limiteront à essayer de cerner le statut de l'image peinte sur les vases. Ce choix est justifié par le fait que l'iconographie céramique possède une véritable spécificité et une véritable indépendance créatrice. On le sait depuis Charles Dugas ${ }^{3}$, mais on ne le répétera jamais assez, l'imagerie des vases peints n'est pas une simple illustration décorative ou anecdotique. Elle est, en elle-même, réflexion et construction de concepts ${ }^{4}$. Elle ne se limite pas à décrire ou à reproduire, mais offre des «modèles comportementaux ${ }^{5}$ » dans diverses activités sociales et notamment dans le domaine religieux. Or cette dimension fondamentale des représentations figurées, considérées comme sources à part entière, a généralement été ignorée ou méconnue dans l'histoire théorique de la religion grecque. Ainsi, le célèbre manuel de Louis Gernet, Le génie grec dans la religion, se contente, dans son chapitre «La religion et l'art», d'envisager la sculpture et la littérature ${ }^{6}$. Mais, en bonne méthode, n'est-il pas imprudent de

2 Quelques exemples parmi d'autres : B. FEHR, Zur religionspolitischen Funktion der Athena Parthenos im Rahmen des delisch-attischen Seebundes, in Hephaistos, 1(1979), p. 71-91; 2(1980), p. 113-125; F. CROISSANT, Art et artisanat dans la plastique attique du $6 e$ s. av. J.-C., in Ktèma, 3(1978), p. 4754; G. SIEBERT, Signatures d'artistes, d'artisans et de fabricants dans l'antiquité classique, ibid., p. 111-132; C. BÉRARD, Le liknon d'Athéna, in $A K, 19(1976)$, p. 101-114; O. DE CAZANOVE, Plastique votive et imagerie dionysiaque, in $M E F \dot{R}, 98(1986)$, p. 7-36. Pour les sources litteraires, cf. l'excellente approche mise en oeuvre par A. SCHNAPP, Why did the Greeks need Images ?, in Third Symposium on Ancient Greek and Related Pottery, Copenhague, 31 août - 4 septembre 1987 (sous presse).

3 C. DUGAS, Décoration et imagerie dans la céramique grecque, in $R E G$, 49(1936), p. 1-25, et Tradition littéraire et tradition graphique, in $A C$, 6(1937), p. 5-26 (articles repris dans le Recueil Charles Dugas, Paris, 1960).

4 Pour une première orientation bibliographique à ce sujet, cf. Cité des images, p. 165 .

5 L'expression est de F. LISSARRAGUE.

6 L. GERNET et A. BOULANGER, Le génie grec dans la religion, Paris, 1932 (2e éd. 1970), surtout p. 196-204. La plupart des grandes maisons d'édition, suivant une habitude néfaste, pratiquent plutôt l'excès contraire et continuent d'accumuler, avec ou sans l'accord des auteurs, des illustrations de toute origine, juxtaposant grandes statues, reliefs, dépôts votifs et vases en tout genre, sans s'interroger sur la valeur exacte de l'information contenue dans chaque image. C'est encore le cas pour le livre de H.W. PARKE, Festivals of the Athenians, Londres, 1977. 


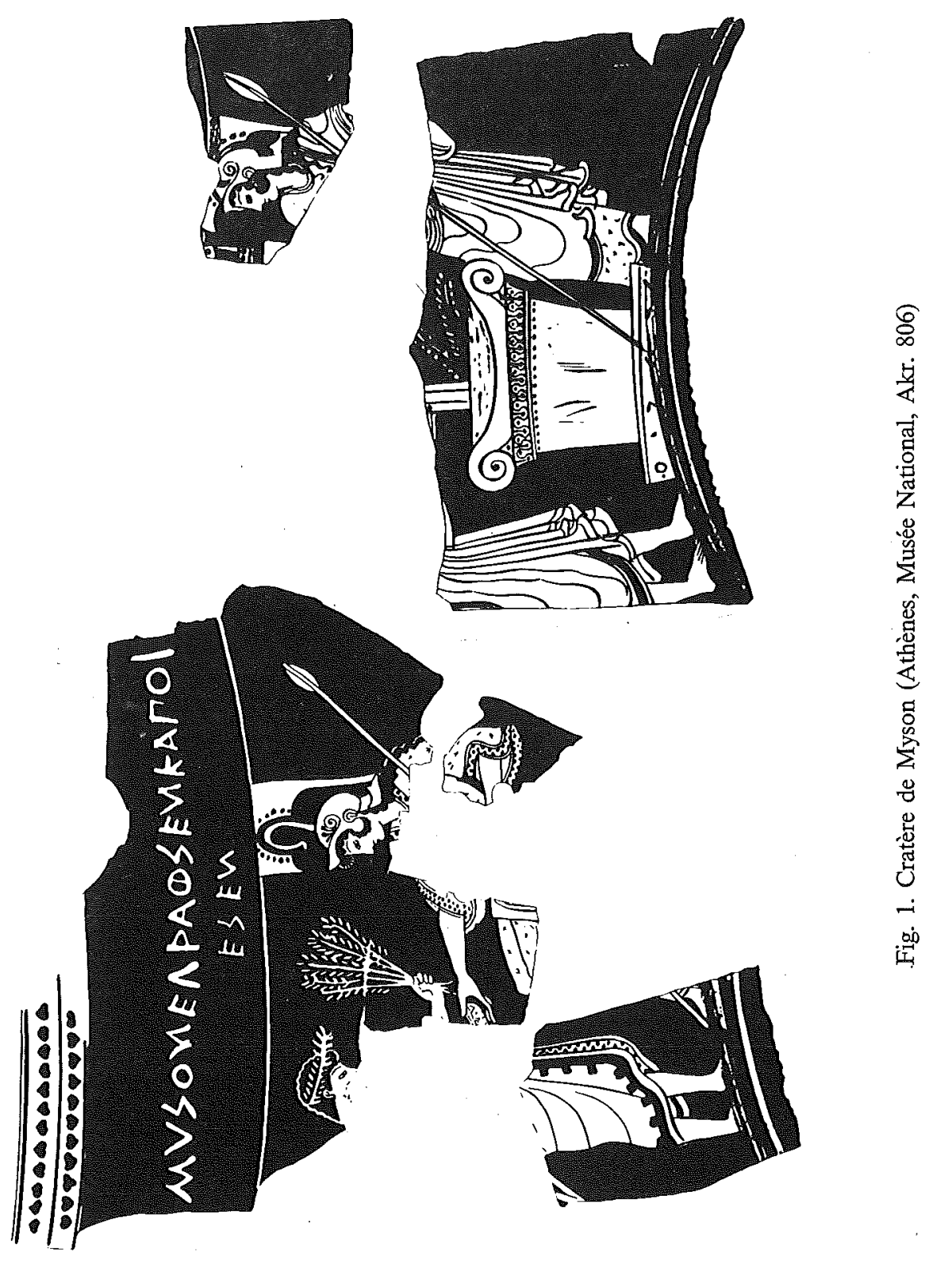




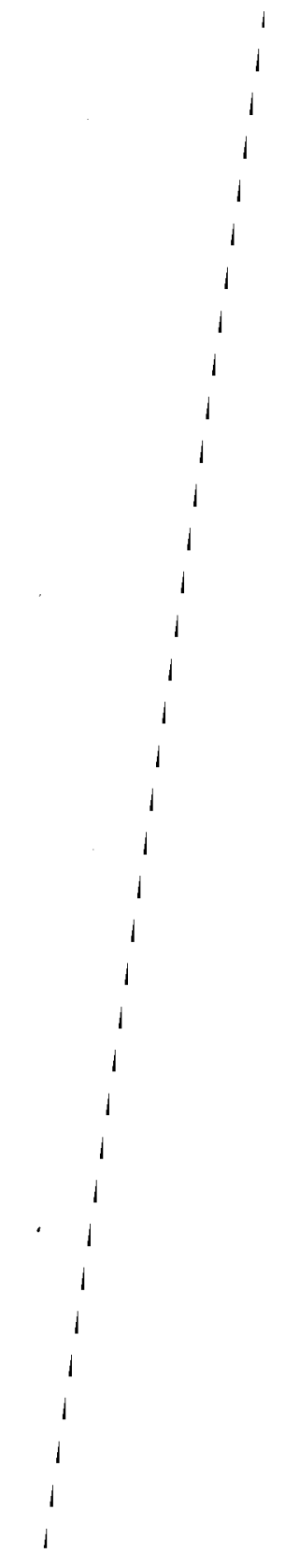


poser, dès le départ, une distinction hiérarchique, qui pourrait bien être moderne, entre un «grand art» chargé de signification, culminant avec la statue de culte, et des «genres mineurs», nécessairement voués aux schémas répétitifs et atypiques ? Quel que soit son support, quelles que soient ses dimensions, chaque image est un message. A nous de le comprendre et d'en évaluer la portée.

Un exemple récent illustre parfaitement cette idée : dans un article consacré aux représentations du sacrifice en Grèce, Folkert van Straten ${ }^{7}$ se propose de comparer les statistiques d'apparition respective du boeuf, du mouton et du porc, non seulement sur les vases attiques à figures noires et à figures rouges d'une part et sur les reliefs votifs d'autre part, mais aussi dans les mentions des calendriers religieux, confrontant ainsi trois types de sources différentes, dont il reconnait et affirme avec prudence les particularités. Ses graphiques révèlent que, dans les calendriers qui énumèrent les sacrifices offerts par une communauté civique ou une association religieuse, généralement au cours d'une phase de réforme ou de restructuration des dépenses, la victime principale est le mouton. Dans les reliefs votifs, offerts par des personnes privées, le porc est le mieux attesté. Dans la céramique, c'est le boeuf qui domine, l'animal le plus cher, le plus glorieux et sans doute le plus rarement sacrifié. Cette première constatation déjà nous alerte : l'imagerie des vases donne une vision particulière, biaisée, de la réalité cultuelle, une vision «rêvée» en quelque sorte, sans que l'on puisse parler ici d'image de compensation ou de substitution...

Après cette première remarque - ou plutôt ce premier avertissement méthodologique, qui sous-tendra l'ensemble de l'exposé -, abordons en quelques grands traits le sujet annoncé.

7 F. VAN STRATEN, Greek Sacrificial Representations : Livestock Prices and Religious Mentality, in Gifts to the Gods. Proceedings of the Uppsala Symposium 1985, Uppsala, 1987 (Boreas, Acta Universitatis Upsaliensis, Uppsala Studies in Ancient Mediterranean and Near Eastern Civilizations, 15), p. 159 170 , en particulier les figures 10-13. 
De façon globale, on peut considérer que l'apport de l'iconographie céramique ${ }^{8}$ en matière d'histoire religieuse concerne deux domaines privilégiés, que nous désignerons ici suivant une terminologie conventionnelle, sans doute discutable dans la mesure où elle introduit un cloisonnement arbitraire, mais commode pour un classement provisoire des données; ces deux domaines sont, d'une part, la «mythologie» et, d'autre part, les aspects matériels du culte, les «realia».

L'étude des représentations mythologiques a suscité une bibliographie déjà énorme, qu'il est impossible de détailler ici : cata$\operatorname{logues}^{9}$ et études thématiques ${ }^{10}$ abondent, que ce soit pour des motifs de grande fréquence ou pour des mythes exceptionnels, rarement illustrés ${ }^{11}$. Notons également la place importante de l'iconographie céramique dans le $L I M C$, répertoire colossal et d'une indéniable utilité, mais dont la réalisation a suscité quelques controverses : dans quelle mesure en effet sommes-nous autorisés à mettre en série les images antiques, suivant des critères modernes ? A partir de quel moment aussi peut-on décider qu'une image est mythologique, puisque l'on sait par ailleurs que les peintres euxmêmes admettaient et créaient l'ambiguïté entre les registres du «réel» et du «mythique»? D'autres tendances novatrices se font jour également dans le domaine de l'iconographie légendaire : ainsi,

8 Pour les orientations actuelles dans l'étude de la céramique grecque, nous renvoyons notamment au Bulletin archéologique. Céramique, que H. METZGER publie régulièrement dans la $R E G$. Cf. aussi les importants colloques récents : Second Symposium on Ancient Greek and Related Pottery, Amsterdam, 11-15 avril 1984, Amsterdam, 1984 et Third Symposium on Ancient Greek and Related Pottery, Copenhague, 31 août - 4 septembre 1987 (sous presse).

9 Par exemple : F. BROMMER, Vasenlisten zur griechischen Heldensage, 3e éd., Marburg, 1973.

10 Une sélection bibliographique assez large apparaît notamment dans les ouvrages de K. SCHEFOLD, Frïhgriechische Sagenbilder, Munich, 1964; Götter - und Heldensagen der Griechen in der sp ätarchaischen Kunst, Munich, 1978; Die Göttersage in der klassischen und hellenistischen Kunst, Munich, 1981; Die Urkönige, Perseus, Bellerophon, Herakles und Theseus in der klassischen und hellenistichen Kunst, Munich, 1987; Die klassischen und hellenistischen Bilder der Sagen von der Argonauten, von Theben und Troja, (sous presse). Le livre de H. METZGER, Les représentations dans la céramique attique du IVe siècle, Paris, 1951, est toujours particulièrement intéressant pour notre propos.

11 Un exemple remarquable a été récemment présenté par H.A. CAHN, Okeanos und Atlas auf einer Spitzamphora, in Third Symposium... (supra n. 8, sous presse). 
la recherche d'une dimension politique ${ }^{12}$, idéologique ${ }^{13}$ ou religieuse ${ }^{14}$.

L'étude des realia paraîtrait a priori plus simple et plus concrète. Mais les difficultés d'interprétation surgissent à chaque pas puisque, pour toute scène figurée, comme pour tout texte, il faut entreprendre une critique interne et externe, tenant compte de multiples facteurs ${ }^{15}$ : outre le sujet, la date précise de l'oeuvre, la forme du vase $^{16}$, le peintre, la provenance, les procédés symboliques et allégoriques... Un exemple suffira à préciser ce point élémentaire. Dans son intéressant ouvrage Potter and Patron in Classical Athens, T.B.L. Webster consacre un chapitre aux scènes cultuelles et réunit, dans un tableau comparatif, les statistiques d'apparition des images dites «de sacrifice» et des images dites «à l'autel»17. Il fait ainsi apparaître une évolution relative, peut-être plus importante à l'analyse qu'elle ne le semble à première vue. En effet, les scènes de procession et de sacrifice collectif, bien attestées en figure noire, subissent au Ve siècle la concurrence des altars scenes des belles coupes à figures rouges de style sévère, où le fidèle est représenté seul devant le bômos. Comme, de toute évidence, il ne s'agit pas ici d'un simple problème technique, mettant en cause par exemple la

12 L'article de J. BOARDMAN, Herakles, Peisistratos and sons, in RA (1972), p. 57-72, est le premier d'une série consacrée à l'importance politique des représentations d'Héraclès dans l'Athènes du VIe siècle. Cf. aussi, du même auteur, $J H S, 95(1975)$, p. 1-12; $A J A, 82(1978)$, p. 11-25; RA (1978), p. 227-234; Second Symposium... (supra n. 8), p. 239-247.

13 Citons par exemple J.M. MORET, L'«apollinisation» de l'imagerie légendaire à Athènes dans la seconde moitié du Se siècle, in RA (1982), p. 109-136.

1.4 Mon article Images et croyances en Grèce ancienne: représentations de l'apothéose d'Héraklès au Ge siècle, in Images et société en Grèce ancienne. Actes du Colloque de Lausanne (supra n. 1), p. 187-199, constitue une tentative en ce sens.

15 A ce titre, l'article de F. LISSARRAGUE, Naples 127929: histoire d'un vase, in $\dot{D} A r c h, 1(1985)$, p. $77-88$, représente un modèle du genre et une excellente leçon de méthode. Pour la nécessité de prudence et de rigueur dans l'observation, puis la description d'une image, cf. tout récemment H. METZGER, Beazley et l'image, in $A K, 30(1987)$, p. 109-118.

16 Par exemple, I. SCHEIBLER, Bild und Gefäss, in JDAI, 102(1987), p. 57118.

17 T.B.L. WEBSTER, Potter and Patron in Classical Athens, Londres, 1972, p. 126-151 et tableau p. 127. Il va de soi que toute forme de statistique relative à la céramique antique doit être utilisée avec la plus grande précaution. Mais elle n'est a priori pas impossible, ni injustifiée sur le plan mathématique. Il s'agit plutôt d'un type de «sondage». 
forme des vases, la question pourrait être posée en termes de mentalité religieuse et il serait justifié de formuler l'hypothèse selon laquelle, au VIe siècle, la piété collective paraît plus importante à montrer que la piété individuelle, en plein avènement de l'époque classique.

La plupart des actes cultuels de la vie religieuse en Grèce antique sont connus par des représentations figurées, principalement grâce aux vases peints. Mais ceux-ci, on le sait, ont leurs centres d'intérêt propres, que nous pouvons suggérer à l'aide de quelques exemples. Ainsi, l'iconographie céramique nous renseigne sans aucun doute sur le déroulement des fêtes, mais pas n'importe lesquelles : les peintres privilégient incontestablement les fêtes d'Athéna et de Dionysos $^{18}$. De même, il faut se garder de toute interprétation abusive, si tentante et satisfaisante que soit l'idée d'une représentation «en direct» de telle ou telle festivité : le cas des beaux stamnoi à figures rouges dits "des Lénéennes" est à cet égard très significatif ${ }^{19}$. L'iconographie céramique nous renseigne aussi sur les gestes et les moments rituels, mais pas de n'importe quelle façon ${ }^{20}$. Pour le thème de la thusia, des recherches récentes ont bien souligné que certaines étapes de la séquence sacrificielle sont volontairement omises de la paroi des vases, et notamment celle de la mise à mort de la victime. Cette observation rejaillit sur la signification générale du sacrifice au coeur de la société classique et sur les controverses modernes relatives à son origine, à sa fonction 21 . Les images de sacrifice sont calmes, construites, harmonieuses ${ }^{22}$.

18 K. LEHNSTAEDT, Prozessionsdarstellungen auf attischen Vasen, Munich, 1970. Les fêtes de Déméter sont également bien attestées. Pour une bibliographie récente, cf. A. MALAGARDIS, Deux temps d'une fête athénienne sur un skyphos attique, in $A K, 28(1985)$, p. 71-92.

19 C. BÉRARD et C. BRON, Bacchos au coeur de la cité, in L'association dionysiaque dans les sociétés anciennes. Actes de la Table Ronde de Rome, Ecole française, 24-25 mai 1984, Rome, 1986 (Collection de l'Ecole française de Rome, 89), p. 13-30, surtout p. 14-15.

20 En Grèce ancienne: images et rituel. Actes de la Table Ronde de l'Arbresle, Centre Thomas More, 24-25 mars 1984, L'Arbresle, 1985 (Recherches et Documents du Centre Thomas More, 48).

21 J.-L. DURAND, Sacrifice et labour en Grèce ancienne, Paris, 1986; Cité des images, p. 49-66.

22 Cf. par exemple le haut degré d'élaboration de la scène principale pour le cratère à figures rouges de Naples 127929, dû au peintre.de Pan : F. LISSARRAGUE, art. cit. (supra n. 15). 
Les «informations» iconographiques peuvent concerner enfin les objets, voire les lieux, du culte. Un somptueux cratère de Ferrare ${ }^{23}$ permet de commenter, dans une certaine mesure, l'usage du thymiatérion ou du kanoûn, mais n'autorise guère à parler de l'aspect du temple ou de la statue cultuelle : les colonnes sont ici des indicateurs d'espace et Apollon, bien que mis à distance des hommes, assiste en personne à la cérémonie... Statues de culte ou dieux présents ? Au fait, la question se pose pour nous, mais pas pour les fidèles. Sur un autre cratère de Ferrare $^{24}$, lui aussi l'un des chefs-d'oeuvre de la figure rouge classique, le couple de divinités assises intervient directement dans l'action rituelle, en effectuant une véritable libation : le peintre a indiqué l'écoulement du liquide. Mais qui sont les dieux en l'honneur desquels s'accomplit cette danse extatique ? Déméter Chloé et un Dionysos chthonien ? Cybèle et Sabazios ? La discussion n'est pas close, mais elle dépasse les limites de notre propos. Nous étions en effet partis de l'idée de présenter l'iconographie comme science auxiliaire de l'étude de la religion grecque et nous risquons d'aboutir à l'étude de la religion grecque comme science auxiliaire de la céramologie. La pluridisciplinarité n'est pas sans paradoxe.

Nous avons évoqué jusqu'ici l'image pour elle-même, hors contexte. Une autre forme d'approche, promise à un avenir certain, consiste à mieux situer l'image dans son contexte archéologique, pour les vases dont la provenance est connue. Nous devons écarter provisoirement, par simple manque de temps, le difficile problème des relations entre artistes grecs et clientèle étrusque ${ }^{25}$; il est exclu d'ignorer que tant de vases attiques répertoriés ont terminé leur carrière dans des tombes d'Italie. Plutôt que de nous engager dans ce large débat, nous focaliserons davantage notre attention sur les tombes et les sanctuaires de la Grèce même, dont on sait avec toute la certitude des fouilles archéologiques qu'ils ont fourni telle ou telle oeuvre.

23 Ferrare, Museo Nazionale, T. 416 B VP, en provenance de Spina, $A R V^{2}, 1144$, 21 = Cité des images, fig. 73.

24 Ferrare, Museo Nazionale, T. 128, en provenance de Spina, $A R V^{2}, 1052,25=$ Cité des images, fig. 21.

25 J. DE LA GENIÈRE, Images attiques et religiosité étrusque, in Third Symposium... (supra n. 8, sous presse). 
Pour l'étude du matériel funéraire, de très intéressantes données ont été révélées par un article remarquable de Lucilla Burn, récemment paru, qui reprend l'étude d'une tombe découverte à Athènes voici près d'un siècle ${ }^{26}$. Il apparaît clairement que les vases choisis pour accompager la défunte forment un ensemble d'une exceptionnelle cohérence, à la fois par la technique et le style, puisqu'ils proviennent de l'atelier de Sotadès, et par l'iconographie, où s'entrecroisent les thèmes de la vie féminine et de l'immortalité, symbolisée par le leitmotiv du miel.

Dans les sanctuaires, les vases, qu'ils soient ou non porteurs d'images, jouent un rôle non négligeable, non seulement en tant qu'objets rituels, mais aussi comme ex-voto. Sur un lécythe à figures rouges de Heidelberg ${ }^{27}$ le peintre a représenté ce qui, à ses yeux, créait un sanctuaire : l'autel, le pilier hermaïque, une colonne à laquelle est suspendue la victime consacrée et, en offrande, une image, partie intégrante de l'espace sacré.

Trois directions de recherche peuvent être proposées à partir des vases provenant de sites religieux. En premier lieu, il serait possible d'étudier cette céramique en établissant des statistiques de fréquence de thèmes figurés, qu'ils soient mythologiques ou autres. De telles monographies, adoptant une méthode uniforme pour chaque site, manquent et toute comparaison, interne ou entre sanctuaires, est encore difficile. Quelques publications isolées, pour Thasos notamment ${ }^{28}$, ont déjà envisagé une telle approche et en ont souligné l'intérêt. Les vases de l'Acropole d'Athènes s'y prêteraient remarquablement, comme le suggère une lecture préliminaire des recueils de B. Graef et E. Langlotz ${ }^{29}$ : si l'on ne s'étonne évidemment pas du succès de la représentation d'Athéna, et en particulier de sa naissance, on peut s'interroger par contre sur la présence envahissante d'Héraclès dans les ex-voto de la déesse. De façon générale, il ne serait sans doute pas inutile de vérifier, pour chaque sanctuaire, si, à côté de nombreux vases à l'iconographie atypique et peut-être même

26 L. BURN, Honey Pots. Three White-Ground Cups by the Sotades Painter, in $A K, 28(1985)$, p. 93-105.

27 Heidelberg, coll. privée, $A R V^{2}, 685,164=$ Cité des images, fig. 86.

28 J.J. MAFFRE, Céramique attique à décor mythologique trouvée à l'Artémision de Thasos, in Thasiaca, Paris, 1979 (BCH Suppl. V), p. 11-74.

29 B. GRAEF et E. LANGLOTZ, Die antiken Vasen von der Akropolis zu Athen, Berlin, 1925-1933. 
«ouverte» ${ }^{30}$, il n'existe pas des séries de vases qui font sens et qui rejoignent ainsi la catégorie du relief votif avec, à la charnière entre ces deux réseaux d'image, la plaque en terre cuite peinte suspendue dans le temenos ${ }^{31}$.

D'autre part, l'étude des dédicaces inscrites ou gravées sur la paroi de certains vases pourrait être entreprise à une large échelle, afin de déterminer s'il s'agit là d'un phénomène isolé ou répandu. Quel est le rapport entre le vase consacré, le dédicant et la divinité ? Un skyphos de Délos ${ }^{32}$ offert à Héra porte une iconographie héracléenne : simple ironie mythographique ou plutôt expression pieuse d'une réalité cultuelle reconnue par le biais de l'image ? Et à quel point le peintre Myson souhaite-t-il s'associer personnellement à la représentation du fidèle d'Athéna, sur son cratère de l'Acropole ${ }^{33}$, le seul de ses vases conservés où son nom apparaisse (fig. 1) ?

Au-delà de ces cas ponctuels où l'inscription peut matérialiser l'existence d'un lien, il faudrait également reprendre de façon systématique toutes les occurrences d'une iconographie en nette relation avec certaines pratiques rituelles ou avec le hieros logos fondateur du sanctuaire ${ }^{34}$. Pensons par exemple à une loutrophore d'Eleusis, à figures noires, qui pourrait figurer, semble-t-il, la translation des sacra ${ }^{35}$. Ou à la majesteuse amphore de Reggio, attribuée à la manière d'Exékias ${ }^{36}$, dont l'iconographie éleusinienne

30 Pour les terres cuites, cf. les commentaires de N. WEILL, Images d'Artémis à

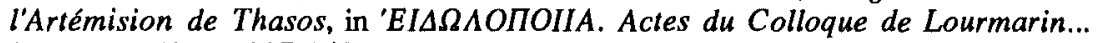
(supra n. 1), p. 137-148.

31 Par exemple, H.A. GEAGAN, Mythological Themes on the Plaques from Penteskouphia, in AA, 85(1970), p. 31-48.

32 Délos, Musée, 595, E. HASPELS, Attic Black-Figured Lekythoi, Paris, 1936, p. 249,3 = C. DUGAS, Les vases de l'Héraion, in Exploration archéologique de Délos X, Paris, 1928, p. 179 et pl. 46.

33 Athènes, Musée National, Akr. 806, $A R V^{2}, 240,42=$ B. GRAEF et E. LANGLOTZ, Die antiken Vasen von der Akropolis zu Athen, II, 2, Berlin, 1931 , pl. 72. Je remercie vivement M. I. Sakellarakis de m'avoir autorisée à reproduire le dessin de ce cratère.

34 Pensons au cas de Brauron: L. KAHIL, L'Artémis de Brauron: rites et mystère, in $A K, 20(1977)$, p. 86-98.

35 Eleusis, Musée, 471 (ex 837), fragments, $A B V, 309,97$ = E. BOEHR, Der Schaukelmaler, Mayence, 1982, p. $100, \mathrm{n}^{\circ} 132$, pl. 136-137. Cf. aussi H. METZGER, Recherches sur l'imagerie athénienne, Paris, 1965, p. 28-29.

36 Reggio, Museo Nazionale, 4001, ABV, 147, 6 = H. METZGER, Recherches... (supra n. 35), pl. I, 2 et II. 
peu habituelle rappelle assez qu'elle fut découverte à Locres, célèbre par son sanctuaire de Perséphone.

Les directions de recherche proposées ici ne sont que de premières ébauches, de banales suggestions. Mais elles ont apparemment un point commun : elles tendent à mettre en évidence l'aspect intentionnel de l'imagerie. Dès lors, ne peut-on se demander jusqu'où va l'intention du dédicant ou, plus généralement, de l'acheteur, en matière religieuse ? Que désire-t-il investir dans le choix d'une image ? Un simple hasard, un vague souvenir ? Ou s'y engage-t-il au point de requérir une véritable protection, non seulement pour lui-même, mais pour l'ensemble de la cité ? A cet égard, les multiples représentations de la Naissance d'Athéna, thème attique par excellence dès les vases du VIe siècle, pourraient être comprises de cette façon aussi ${ }^{37}$. En poursuivant ce raisonnement jusqu'à l'excès, on en arriverait même à se poser la question de savoir si certains vases grecs ont pu jouer le rôle de ces innombrables images pieuses qui circulaient dans plusieurs pays d'Europe occidentale au cours des XVIIIe et XIXe siècles ${ }^{38}$. Or ces images pieuses, au sens saint-sulpicien du terme, ne manquent pas de ressemblances, évidemment fortuites, avec l'iconographie céramique de la Grèce ancienne : la matière est peu noble (bois, papier) mais vivement colorée; la scène, à valeur narrative, exalte un moment édifiant de l'histoire du saint, identifié par ses attributs; didactique, mais surtout apotropaïque, la vertu conférée à ces tableaux incitait les fidèles à les placer entre leurs vêtements ou sous leur oreiller; on allait même jusqu'à découper l'image et à la mélanger à la nourriture d'un malade pour accélérer la guérison ${ }^{39}$. Ingérer l'image: c'est sans doute là un des stades extrêmes de l'impact religieux d'une représentation pourtant modeste...

37 Renvoyons par exemple à N. LORAUX, Les enfants d'Athéna, Paris, 1981.

38 Pour ce thème, MM. B. Fornari et Y. Quairiaux m'ont généreusement procuré de multiples informations. Qu'ils soient ici vivement remerciés pour cette aide indispensable et amicale. Cf. en particulier R. DARQUENNE, Y. QUAIRIAUX et al., Médecine et société. Catalogue de l'exposition organisée par le Musée royal de Mariemont, 27 mars - 30 août 1987, Morlanwelz, 1987, surtout p. 153-158; J. PIROTTE, Images des vivants et des morts. La vision du monde propagée par l'imagerie de dévotion dans le Namurois, 1840-1965, Louvain-la-Neuve, 1987.

39 R. COOLEN, La médecine populaire. Catalogue de l'exposition organisée à Bruxelles, Galerie CGER, 27 mars-17 mai 1981, Bruxelles, 1981, nº 166. 
Cette comparaison, volontairement outrancière, est hors de propos pour la religion grecque antique, sans dogme, sans iconoclasme $^{40}$. Le but de ce faux parallèle était de susciter une réaction, peut-être salutaire, et d'inviter à porter un autre regard sur l'imagerie des vases grecs qui, semble-t-il, pouvaient se situer sur différents plans de sacralité. Trois exemples sélectionnés parmi bien d'autres permettront de conclure. Une hydrie à figures noires d'Uppsala ${ }^{41}$ ne tente-t-elle pas de traduire l'émoi religieux suscité par l'épiphanie de la déesse ? Les nombreuses représentations du Festin d'Héraclès, en figure noire et en figure rouge, ne se contentent peut-être pas de dire le repos du héros entre deux exploits, mais semblent davantage exalter le triomphe du dieu au canthare, tel qu'il apparaît au coeur de ses sanctuaires. Enfin, il serait légitime d'admettre que, sur le célèbre cratère de New York, attribué au peintre de Perséphone ${ }^{42}$, l'image dise le mythe, enseigne le mythe, au même titre que la tradition orale des Hymnes et avec la même force.

Image-protection, image-référent, image-espoir ? Autant de possibilités, autant d'interrogations encore sans réponse. Mais la mission du C.E.R.G.A. n'est-elle pas, précisément, de bien poser, sinon de résoudre, de nouvelles questions dans le domaine de la religion grecque, dont l'étude nous réunit à Athènes ?

Annie VERBANCK-PIÉRARD

Musée royal de Mariemont B - 6510 MORLANWELZ

40 Cf. les propos d'A. SCHNAPP, art. cit. (supra n. 2).

41 Uppsala, Gustavianum, 352, $A B V, 519,15=$ Cité des images, fig. 154. C. MELLDAHL et J. FLEMBERG, Eine Hydria des Theseus-Malers mit einer Opferdarstellung, in From the Gustavianum Collections in Uppsala, 2, 1978. The Collection of Classical Antiquities, Uppsala, 1978 (Boreas. Acta Universitatis Upsaliensis, Uppsala Studies in Ancient Mediterranean and Near Eastern Civilizations, 9), p. 57-79.

42 New York, Metropolitan Museum, 28.57.23, ARV $V^{2}, 1012,1=$ Cité des images, fig. 159. 